\title{
Computational Study of Mixed Convection Flow with Algebraic Decay of Mainstream Velocity in the Presence of Applied Magnetic Field
}

\author{
Muhammad Ashraf*, Uzma Ahmad, Masud Ahmad and Sultana N
}

Department of Mathematics, Faculty of Science, University of Sargodha, Pakistan

\begin{abstract}
The present study concern with the two dimensional viscous, incompressible, electrically con ducting, mixed convection flows with algebraic decay of mainstream velocity $U(x)=(1-x)^{-a}$. The physical phenomenon of the problem is simulated by using the Primitive Variable Formulation (PVF) for Finite Difference Method (FDM) and Stream Function Formulation (SFF) for Local Non-Similarity Method (LNS). The physical behaviors of momentum and temperature fields are given graphically. The results obtained for skin friction and rate of heat transfer for different values of physical parameters are also compared by both methods and presented in tabular form.
\end{abstract}

Keywords: Mixed convection; Heat transfer; Skin friction; Algebraic decay of mainstream velocity

\section{Introduction}

When the surface temperature is different from the ambient fluid temperature, it gives rise to a density gradient in the thermal boundary layer region. This density gradient produces a body force in the form of buoyancy of the fluid. The buoyancy force along with some external force constitutes a mixed convection flow. There the external force can either be in the form of free stream or some moving or stretching surface. With this understanding, we formulate the problem of mixed convection flow and the possible literature survey for the problem under consideration is given in preceding paragraph.

Ostrach [1] presented the similarity solution of natural convection along vertical isothermal plate. The instantaneous action of buoyancy and induced magnetic forces leading to natural convection heat transfer was investigated by Sparrow et al. [2]. They found out that in the presence of magnetic field, the natural convection heat transfer to liquid metals may be considerably affected [3]. The mixed convection over a horizontal surface due to uniform free stream has been studied by many researchers. Gebhart [4] used an analytical technique to analyze the effect of dissipation on natural convection $[5,6]$. The joint free and forced convection along with uniform heat flux in the presence of strong magnetic field was studied by Lioyd el al. [7]. Dwivedi [8] examined the free convection oscillatory hydromagnetic laminar flow of a viscous, electrically conducting and incompressible fluid over a vertical infinite porous plate under the influence of a transverse magnetic field when the temperature or heat flux at the surface oscillates in magnitude but not in direction. Soundalgekar [9] studied natural convection flow along vertical porous plate with suction and viscous dissipation [10].

A particular self-similar solution of the mixed convection flow over a horizontal surface was presented by Schneider [11] for the case of specified wall temperature as an inverse square root of the distance from the top edge. Chakrobarti et al. [12] illustrated the heat transfer and flow of an incompressible electrically conducting fluid pass a stretching sheet. They presented an analytical solution for the flow and the numerical solution for the heat transfer. Under the influence of free stream oscillations, Perdikis et al. [13] examined the laminar free convection and mass transfer effects of a viscous, incompressible heat generating fluid-flow along an impulsively vertical plate with heat flux and constant suction at the plate. Wantanabe et al. [14] investigated the heat transfer in thermal boundary layer of MHD flow over a flat plate. The mixed convection boundary layer flow of an incompressible, viscous and electrically conducting fluid in the presence of transverse magnetic field along a vertical porous plate was examined by Hosain et al. [15]. In the presence of Hall current effect, Chamkha [16] expressed the MHD free convection pass a vertical plate in thermally satisfied porous medium. Chen [17] presented his study on MHD natural convection flow over a permeable inclined surface with variable wall temperature and concentration. He studied that increasing the angle of inclination decreases the effect of buoyancy force. Mostafa [18] studied the heat transfer and flow of an incompressible electrically conducting viscous fluid which passes a continuously moving vertical infinite plate with uniform suction and heat flux under the action of radiation taking into account the effects of variable viscosity. Considering a homogeneous chemical reaction of first order, Odat et al. [19] numerically examined the effect of magnetic field on the transient free convection flow of an electrically conducting fluid pass an impulsively started isothermal vertical plate. They observed that increasing the chemical reaction parameter decreases the velocity and concentration. Makinde [20] performed an analysis to examine the hydromagnetic mixed convection flow of a viscous, incompressible and electrically conducting fluid and mass transfer along a vertical porous plate with constant heat flux embedded in a porous medium.

However, when the free convective flows occur at high temperatures, radiation effects on the flow become significant. Many processes in engineering areas occur at high temperatures and knowledge of radiative heat transfer becomes very important for the design of the pertinent equipment. Nuclear power plants, gas turbines and the various propulsion devices for aircraft, missiles and space vehicles are examples of such engineering areas. The inclusion of radiation effects in the energy equation leads to a highly non-linear partial differential equation. Under the influence of thermal radiation along with variable

*Corresponding author: Muhammad Ashraf, Department of Mathematics, Faculty of Science, University of Sargodha, Pakistan, Tel: 9248 9230811; E-mail: mashraf682003@yahoo.com

Received June 20, 2015; Accepted July 07, 2015; Published July 13, 2015

Citation: Ashraf M, Ahmad U, Ahmad M, Sultana N (2015) Computational Study of Mixed Convection Flow with Algebraic Decay of Mainstream Velocity in the Presence of Applied Magnetic Field. J Appl Mech Eng 4: 175. doi:10.4172/2168 9873.1000175

Copyright: $\odot 2015$ Ashraf M, et al. This is an open-access article distributed under the terms of the Creative Commons Attribution License, which permits unrestricted use, distribution, and reproduction in any medium, provided the original author and source are credited. 
suction and thermophoresis. Alam et al. [21] numerically examined the two-dimensional steady MHD mixed convection and mass transfer flow through an inclined semi-infinite porous plate. In the presence of transversely applied magnetic field, MHD flow of an electrically conducting, viscous and incompressible fluid through a semi-infinite vertical plate with mass transfer was examined by Palani et al. [22]. Ferdows et al. [23] presented MHD boundary layer flow of a nanofluid through an exponentially stretching sheet. They also discussed and observed the heat transfer characteristics and the effects of governing parameters on the flow field. Ashraf et al. [24] studied the mixed convection flow past a magnetized vertical porous plate numerically.

In the light of above literature survey, we formulate the problem of mixed convection flow with algebraic decay of mainstream velocity in the presence of applied magnetic field.

\section{Mathematical Analysis and Governing Equations}

In this section, we formulate the bulk of mathematical equations governing the MHD convective flow along a vertical flat plate. We combine the basic momentum and energy equations into self-consistent system. This set of equations considers steady two dimensional flow of viscous incompressible conducting fluid along a semi-infinite vertical surface. The decay is only algebraic for $0<\alpha<1$ (Figure 1). A magnetic field of strength $B o(x)$ is considered to be applied parallel to the $y$-axis which is normal to the plate. Under the usual Boussinesq approximation, the flow is governed by the following boundary layer equations following by Merkin [10].

$$
\begin{aligned}
& \frac{\partial u}{\partial x}+\frac{\partial v}{\partial y}=0 \\
& u \frac{\partial u}{\partial x}+v \frac{\partial v}{\partial y}=U \frac{d U}{d x}+v \frac{\partial^{2} u}{\partial y^{2}}+g \beta\left(T-T_{\infty}\right)-\frac{\sigma B_{0}^{2}}{\rho} u \\
& u \frac{\partial T}{\partial x}+v \frac{\partial T}{\partial y}=\alpha \frac{\partial^{2} T}{\partial y^{2}}
\end{aligned}
$$

where

$$
U(x)=(1-x)^{-\alpha}
$$

The appropriate boundary conditions for the present problem are

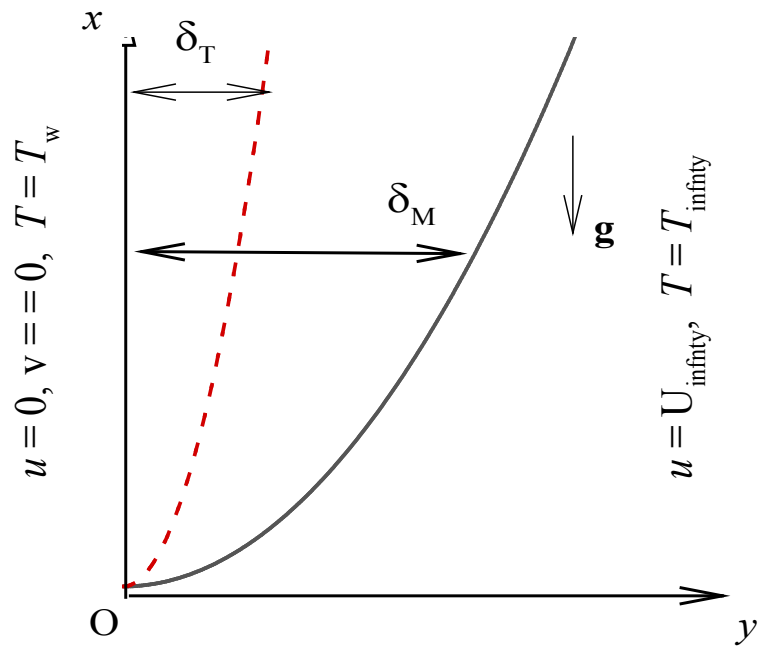

Figure 1: Boundary layer equations.

$$
\begin{aligned}
& u(x, 0)=0, \quad v(x, 0)=0, \quad T(x, 0)=T_{w} \\
& u(\infty)=U(x), \quad T(\infty)=T_{\infty}
\end{aligned}
$$

By using the dimensionless variables

$$
\begin{aligned}
& \bar{x}=\frac{x}{L}, \quad \bar{y}=R_{e x}^{\frac{1}{2}} \frac{y}{L}, \quad \bar{u}=\frac{u}{U_{0}}, \quad \bar{v}=R_{e x}^{\frac{1}{2}} \frac{v}{U_{0}} \\
& \bar{\theta}=\frac{\bar{T}-\bar{T}_{\infty}}{\bar{T}_{w}-\bar{T}_{\infty}}, \quad \bar{U}=\frac{U}{U_{0}}
\end{aligned}
$$

The dimensionless boundary layer equations along with boundary conditions are given as:

$$
\begin{aligned}
& \frac{\partial \bar{u}}{\partial \bar{x}}+\frac{\partial \bar{v}}{\partial \bar{y}}=0 \\
& \bar{u} \frac{\partial \bar{u}}{\partial \bar{x}}+\bar{v} \frac{\partial \bar{u}}{\partial \bar{y}}=\bar{U} \frac{d \bar{U}}{d \bar{x}}+\frac{\partial^{2} \bar{u}}{\partial \bar{y}^{2}}+\lambda \bar{\theta}-M \bar{u} \\
& \bar{u} \frac{\partial \bar{\theta}}{\partial \bar{x}}+\bar{v} \frac{\partial \bar{\theta}}{\partial \bar{y}}=\frac{1}{P_{r}} \frac{\partial^{2} \bar{\theta}}{\partial \bar{y}^{2}}
\end{aligned}
$$

The dimensionless boundary conditions are:

$$
\begin{aligned}
& \bar{u}(x, 0)=0, \quad \bar{v}(x, 0)=0, \quad \bar{\theta}(x, 0)=1 \\
& \bar{u}(\infty)=\bar{U}(x), \quad \bar{\theta}(\infty)=0
\end{aligned}
$$

where

$$
\lambda=\frac{G_{r L}}{R_{e L}^{2}}, \quad G_{r L}=\frac{g \beta \Delta T L^{3}}{v^{2}}, \quad R_{e L}=\frac{U_{0} L}{v}, \quad M=\frac{\sigma B_{0}^{2} x^{-1}}{\rho U_{0}}, \quad P_{r}=\frac{v}{\alpha}
$$

the Richardson Grashof number, Reynolds number, magnetic parameter, Prandtl number and the characteristic length respectively.

\section{Method of Solutions}

We now turn to get the numerical solutions of the problem. For this purpose we will use Primitive variable formulation for Finite Difference Method and Stream function formulation for Local NonSimilarity Method.

\section{Primitive variable formulation}

We now introduce the group of transformations that is known as Primitive Variable Formulation (PVF) to transform the given system of equations into convenient form for integration

$$
\begin{aligned}
& \bar{u}=U(\xi, Y), \quad \bar{v}=\bar{x}^{\frac{1}{2}} V(\xi, Y), \quad \bar{U}=(1-\bar{x})^{-\alpha} \\
& \bar{\theta}=\bar{x}^{-1} \theta(\xi, Y), \quad Y=\bar{x}^{-\frac{1}{2}} \bar{y}, \quad \xi=-\bar{x}
\end{aligned}
$$

Thus (5) and (6) becomes

$$
\begin{aligned}
& U \xi \frac{\partial U}{\partial \xi}-\left(\frac{1}{2} Y U+V \xi\right) \frac{\partial U}{\partial Y}=\frac{\partial^{2} U}{\partial Y^{2}}+\lambda \theta-M U-\alpha \xi(1+\xi)^{-2 \alpha-1} \\
& U \xi \frac{\partial U}{\partial \xi}-\left(\frac{1}{2} Y U+V \xi\right) \frac{\partial \theta}{\partial Y}-U \theta=\frac{1}{P_{r}} \frac{\partial^{2} \theta}{\partial Y^{2}}
\end{aligned}
$$

The appropriate boundary conditions satisfied by the above system of equations are

$$
U(\xi, 0)=V(\xi, 0)=0, \quad \theta(\xi, 0)=1
$$


$V(\xi, \infty)=1, \quad \theta(\xi, \infty)=0$

Discretization: Now we first discretize (9)-(20) using FDM (Finite Difference Method)

$$
\begin{aligned}
& \frac{\partial U}{\partial \xi}=\frac{U_{i, j}-U_{i, j+1}}{\Delta \xi} \\
& \frac{\partial U}{\partial Y}=\frac{U_{i+1, j}-U_{i-1, j}}{2 \Delta Y} \\
& \frac{\partial^{2} U}{\partial Y^{2}}=\frac{U_{i+1, j}-2 U_{i, j}+U_{i-1, j}}{\Delta Y^{2}}
\end{aligned}
$$

and get discretized form

$$
A_{1} U_{i+1, j}-B_{1} U_{i, j}+C_{1} U_{i-1, j}=D_{1}
$$

where

$$
\begin{aligned}
& A_{1}=1+\frac{1}{2} \Delta Y\left(\frac{1}{2} Y_{j} U_{i, j}+V_{i, j} \xi_{i}\right) \\
& B_{1}=2+\frac{\Delta Y^{2}}{\Delta \xi} U_{i, j} \xi_{i}+M \Delta Y^{2} \\
& C_{1}=1-\frac{1}{2} \Delta Y\left(\frac{1}{2} Y_{j} U_{i, j}+V_{i, j} \xi_{i}\right) \\
& D_{1}=-\frac{\Delta Y^{2}}{\Delta \xi} U_{i, j} \xi_{i} U_{i, j+1}-\lambda \theta_{i, j} \Delta Y^{2}+\alpha \xi_{i}\left(1+\xi_{i}\right)^{-2 \alpha-1} \Delta Y^{2}
\end{aligned}
$$

Similarly we discretize (10) using FDM

$$
\begin{aligned}
& \frac{\partial \theta}{\partial \xi}=\frac{\theta_{i, j}-\theta_{i, j+1}}{\Delta \xi} \\
& \frac{\partial \theta}{\partial Y}=\frac{\theta_{i+1, j}-\theta_{i-1, j}}{2 \Delta Y} \\
& \frac{\partial^{2} \theta}{\partial Y^{2}}=\frac{\theta_{i+1, j}-2 \theta_{i, j}+\theta_{i-1, j}}{\Delta Y^{2}}
\end{aligned}
$$

and get the discretized form

$$
A_{2} \theta_{i+1, j}-B_{2} \theta_{i, j}+C_{2} \theta_{i-1, j}=D_{2}
$$

where

$$
\begin{aligned}
& A_{2}=\frac{1}{P_{r}}+\frac{1}{2} \Delta Y\left(\frac{1}{2} Y_{j} U_{i, j}+V_{i, j} \xi_{i}\right) \\
& B_{2}=-+-U_{i, j}+\Delta Y U_{i^{\prime}, j} \\
& C_{2}=\frac{1}{P_{r}}-\frac{1}{2} \Delta Y\left(\frac{1}{2} Y_{j} U_{i, j}+V_{i, j} \xi_{i}\right) \\
& D_{2}=-\frac{\Delta Y^{2}}{\Delta \xi} U_{i, j} \xi_{i} \theta_{i, j+1}
\end{aligned}
$$

From this, we get a tri-diagonal system of algebraic equations, which is solved by using Gaussian elimination technique. The computational domain is of size $80 \times 80$ mesh points, and computational is started from $\xi=0$ and march down implicitly, for this we have taken the step size $\Delta \xi=0.05$ and $\Delta Y=0.05$. We calculate the effects of different parameters on coefficients of skin friction, rate of heat transfer, velocity and temperature field with the help of equations.

$$
R e_{x}^{\frac{1}{2}} C f_{x}=\left(\frac{\partial U}{\partial y}\right)_{Y=0}, N u_{x} \operatorname{Re}_{x}^{-\frac{1}{2}}=\left(\frac{\partial \theta}{\partial Y}\right)_{Y=0}
$$

and velocity can be calculated directly using equation of continuity (4) as shown below:

$$
V_{i, j}=V_{i-1, j}-\frac{1}{2}\left(\xi \frac{\Delta y}{\Delta \xi}-Y_{j}\right) U_{i, j}+\frac{1}{2} \xi \frac{\Delta y}{\Delta \xi} U_{i, j-1}-\frac{1}{2} Y_{j} U_{i-1, j}
$$

\section{Stream function formulation}

To get the set of equations in convenient form for integration, we define the following one parameter of transformations for the dependent and independent variables.

$$
\begin{aligned}
& \psi=(2 x)^{\frac{1}{2}}(1-x)^{\frac{1}{2}(1-\alpha)} G(x, \eta) \\
& \eta=\frac{y}{(2 x)^{\frac{1}{2}}(1-x)^{\frac{1}{2}(1-\alpha)}} \\
& \theta=\frac{T-T_{\infty}}{T_{w}-T_{\infty}}
\end{aligned}
$$

By using this group of transformations we have $x$ and $y$ components of velocity as

$$
\begin{aligned}
& u=(-x) \quad G^{\prime} \\
& v=-\frac{(2 x)^{\frac{1}{2}}(1-x)^{\frac{1}{2}(1-\alpha)}}{2 x(1-x)}\left(2 x(1-x) \frac{\partial G}{\partial x}+\eta(2 x+\alpha x-1) G^{\prime}-x(1-\alpha) G+(1-x) G\right)
\end{aligned}
$$

Now the complete transformed form of the model of the problem attains the following form

$$
\begin{aligned}
& G^{\prime \prime \prime}+2 \alpha x\left(1-G^{2}\right)+2(1-x) \lambda \theta-2 M\left(G^{\prime}-1\right)+(1-2 x+\alpha x) G G^{\prime \prime} \\
& =2 x(1-x)\left(G^{\prime} \frac{\partial G^{\prime}}{\partial x}-G^{\prime \prime} \frac{\partial G}{\partial x}\right) \\
& \frac{1}{P_{r}} \theta^{\prime \prime}-\theta^{\prime} G(1-2 x+\alpha x)=2 x(1-x)\left(G^{\prime} \frac{\partial \theta}{\partial x}-\theta^{\prime} \frac{\partial G}{\partial x}\right)
\end{aligned}
$$

with transformed boundary conditions

$$
\begin{aligned}
& G(0)=0, \quad G^{\prime}(0)=0, \quad \theta(0)=1 \\
& G^{\prime}(\infty)=1, \quad \theta(\infty)=0
\end{aligned}
$$

\section{Local non-similarity method}

The solution of the set of locally non-similar equations can be obtained by using local Non- similarity method (LNS). The governing coupled Partial Differential equations are transformed into a sequence of coupled ordinary differential equations (ODE), which are then solved numerically. The local non-similarity method avoids this by deleting the stream-wise a derivative term, that is, the term with $\partial / \partial \xi$. This changes the partial differential equation (PDE) into non-linear ODE, that still has to be solved numerically. The local non-similarity method was first developed by Sparrow and Yu [6], Minkowycz and Sparrow [2] and later used by Chen [17] in his work.

To adopt this method, we first retain all terms in the transformed form with $x$ derivative. Now the systems of equations that we have to 
solve by using LNS are equations (23) and (24).

Now by putting $F=\partial G / \partial x$ and $\varphi=\partial \theta / \partial x$ we have:

$G^{\prime \prime \prime}+2 \alpha x\left(1-G^{2}\right)+(1-2 x+\alpha x) G G^{\prime \prime}+2 \lambda(1-x) \theta+2 M\left(1-G^{\prime}\right)=2 x(1-x)\left(G^{\prime} F^{\prime}-G^{\prime \prime} F\right)$

$\frac{1}{P_{r}} \theta^{\prime \prime}-(1-2 x+\alpha x) G \theta^{\prime}=2 x(1-x)\left(G^{\prime} \varphi-\theta^{\prime} F\right)$

With boundary conditions

$$
\begin{aligned}
& F(0)=0, \quad F^{\prime}(0)=0, \quad \varphi(0)=1 \\
& F^{\prime}(\infty)=1, \quad \varphi(\infty)=0 \\
& F^{\prime \prime \prime}-4 \alpha x G^{\prime} F^{\prime}+2 \alpha\left(1-G^{\prime 2}\right)+(1-2 x+\alpha x)\left(G F^{\prime \prime}-G^{\prime \prime} F\right) \\
& +(\alpha-2) G G^{\prime \prime}+2 \lambda(1-x) \varphi-2 \lambda \theta=2 x(1-x)\left(F^{\prime 2}-F F^{\prime \prime}\right)+2(1-2 x)\left(G^{\prime} F^{\prime}-G^{\prime \prime} F\right) \\
& \frac{1}{P_{r}} \varphi^{\prime \prime}-(1-2 x+\alpha x)\left(G \varphi^{\prime}+\theta^{\prime}\right)-(\alpha-2) G \theta^{\prime}=2 x(1-x)\left(\varphi F^{\prime}-F \varphi^{\prime}\right)+2(1-2 x)\left(G^{\prime} \varphi-\theta^{\prime} F\right)
\end{aligned}
$$

With boundary conditions

$$
\begin{aligned}
& F(0)=0, \quad F^{\prime}(0)=0, \quad \varphi^{\prime}(0)=1 \\
& F^{\prime}(\infty)=1, \quad \varphi(\infty)=0
\end{aligned}
$$

This is the second level of truncation of the Local Non-Similarity because this approximation is made by dropping terms in second level of equations. This system of coupled nonlinear ordinary differential equation now can be solved numerically by employing the Nachtsheim-Swigert [5] iteration technique in conjunction with the shooting procedure to determine the unknown boundary conditions $f^{\prime \prime}(0)$ and $\theta^{\prime}(0)$. Once we find the unknowns $f^{\prime \prime}(0)$ and $\theta^{\prime}(0)$, we are able to find the coefficients of skin friction and rate of heat transfer for different effects of parameters such as algebraic decay parameter $\alpha$, Hartmann number $M$, Richardson parameter $R i$ and Prandtl number Pr with the help of following relations:

$$
R e_{x}^{\frac{1}{2}} C f_{x}=f^{\prime \prime}(\xi, 0), \quad N u_{x} \operatorname{Re}_{x}^{-\frac{1}{2}}=\theta^{\prime}(\xi, 0)
$$

\section{Results and Discussions}

In this section we will discuss the results obtained by Finite Difference Method and Local Non- Similarity Method. In this study, we explore the effects of different parameters on coefficients of skin friction, rate of heat transfer, velocity field and magnetic field. With this understanding the Figures $2 \mathrm{a}$ and $2 \mathrm{~b}$ presents the effects of different values of algebraic decay parameter $\alpha=0.01,0.05,0.1$ by keeping other parameters constant on momentum and boundary layer thicknesses. From these figures it is possible to see how the algebraic decay parameter
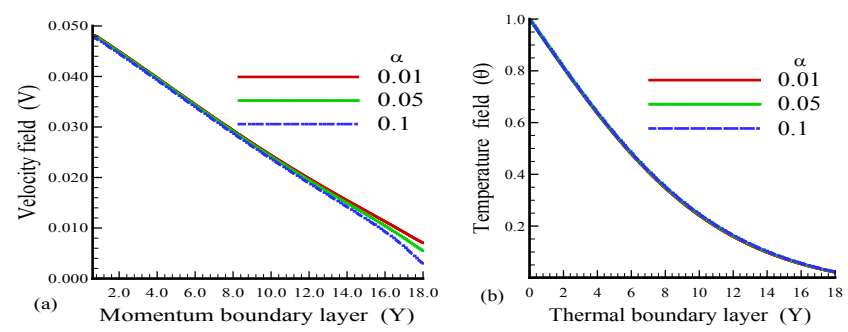

Figure 2: (a) velocity and (b) temperature field profile against boundary layer thickness $Y$ for different values of algebraic decay parameter $\alpha=0.01,0.05$, 0.10 when $P_{r}=0.71, M=1.0$ and for Richardson number $R_{i}=1.0$.
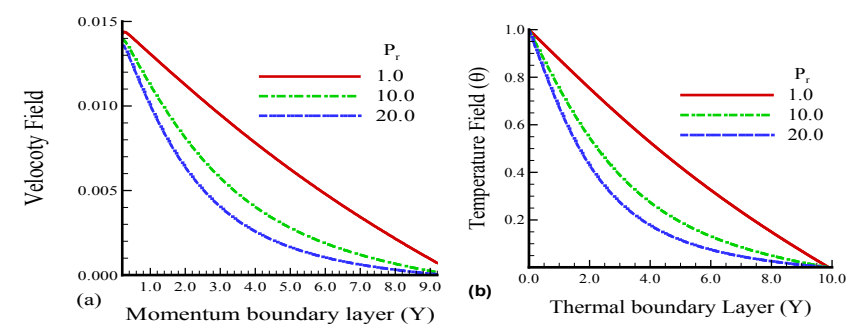

Figure 3: (a) velocity and (b) temperature field profile against boundary layer thickness $Y$ for different values of Prandtl number $P=1.0,10.0,20.0$ when $\alpha=0.07 M=1.0$, and for Richardson number $R_{i}=5.0$.
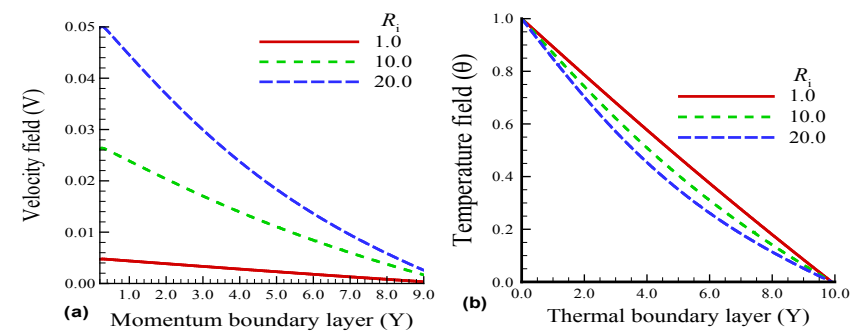

Figure 4: (a) velocity and (b) temperature field profile against boundary layer thickness $Y$ for different values of Richardson number $R_{i}=1.0,10.0,20.0$ when $\alpha=0.07 M=1.0$, and for Prandtl number $P_{r}=0.71$.

$\alpha$ influences the free stream velocity distribution at $Y=16-18$, and there is no effects of algebraic decay parameter on temperature distribution. The reason is that if we see the equation (2) the algebraic decay is only the part of free stream velocity $U=(1-x)-\alpha$ outside the boundary so in both cases this parameter has its no effects within the boundary layer.

In Figures 3a and 3b, the influence of Prandtl number Pr (which is the ratio of kinematic viscosity to thermal diffusion) on momentum and thermal boundary layer thicknesses is shown. For increasing value of Prandtl number both the momentum and thermal boundary layer thicknesses are decreased with the reason that with the increase of Prandtl number Pr the fluid become more viscous which slow down the fluid motion, so distributions on both cases are decaying and this decay is asymptotic. Figures $4 \mathrm{a}$ and $4 \mathrm{~b}$ show some results of variation of Richardson/mixed convection parameter $R_{i}=1.0,10.0,20.0$ on velocity and temperature profile. In this range of Richard parameter the momentum boundary layer thickness is decreased because the Richardson number acts like pressure gradient which take the responsibility of this physical phenomena but its effects in the case of thermal boundary layer is vice versa. Figures $5 \mathrm{a}$ and $5 \mathrm{~b}$ shows the behavior of various values of Hartmann number $M=1.0,10.0,20.0$ on momentum and thermal boundary layer thicknesses. From this numerical exercise we can see the increase of applied magnetic field momentum boundary layer is decreased and thermal boundary layer is increased for maximum value of $\mathrm{M}=20.0$.

Tables 1 and 2 shows some of the results obtained by Finite Difference Method and Local Non-Similarity method for different values of Hartmann number $M$ on coefficient of skin friction and rate of heat transfer. From these tables, it is conclude that with the increase of Hartmann number $M$ the coefficient skin friction and rate of heat transfer both are decreased. For increasing value of Hartmann number increase the magnetic energy and decrease the kinetic energy in the fluid flow domain due to this reason both quantities are decreased. 

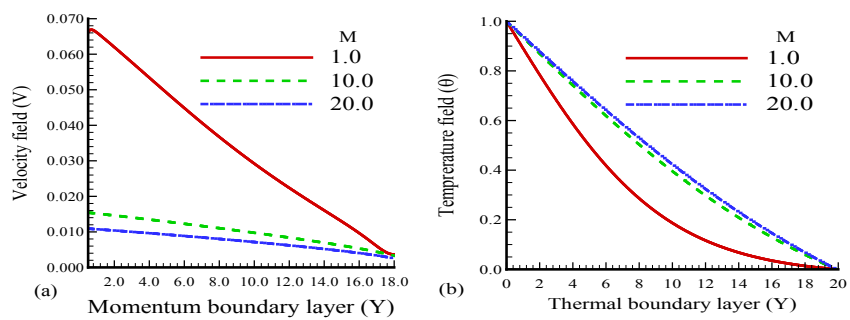

Figure 5: (a) velocity and (b) temperature field profile against boundary layer thickness $Y$ for different values of Hartman number $M=1.0,10.0,20.0$ when $\alpha=0.07 R_{i}=1.0$ and for Prandtl number $P_{r}=0.71$.

\begin{tabular}{|c|c|c|}
\hline $\mathbf{M}$ & FDM & LNS \\
\hline 0.1 & 0.79478 & 0.79104 \\
\hline 1.0 & 0.47204 & 0.47975 \\
\hline 2.0 & 0.39717 & 0.37143 \\
\hline 3.0 & 0.35781 & 0.34295 \\
\hline 4.0 & 0.33172 & 0.33344 \\
\hline 5.0 & 0.29738 & 0.29370 \\
\hline 6.0 & 0.28504 & 0.27167 \\
\hline 7.0 & 0.27465 & 0.25359 \\
\hline 8.0 & 0.26571 & 0.22936 \\
\hline
\end{tabular}

Table 1: Numerical values of coefficient of Skin friction $\operatorname{Re}_{x}^{\frac{1}{2}} C f_{x}$ obtained for different values of $M$ when $P_{r}=10.0, \alpha=0.4, R_{i}=2.0$.

\begin{tabular}{|c|c|c|}
\hline $\mathbf{M}$ & FDM & LNS \\
\hline 0.1 & 0.58954 & 0.58320 \\
\hline 1.0 & 0.36669 & 0.36761 \\
\hline 2.0 & 0.31328 & 0.31274 \\
\hline 3.0 & 0.28548 & 0.27415 \\
\hline 4.0 & 0.26716 & 0.26240 \\
\hline 5.0 & 0.25387 & 0.23197 \\
\hline 6.0 & 0.24357 & 0.23177 \\
\hline 7.0 & 0.223526 & 0.21319 \\
\hline 8.0 & 0.22834 & 0.20981 \\
\hline
\end{tabular}

Table 2: Numerical values of coefficient of rate of heat transfer $N u_{x} R e_{x}^{-\frac{1}{2}}$ obtained for different values of $M$ when $P_{r}=10.0, \alpha=0.4, R_{i}=2.0$.

\begin{tabular}{|c|c|c|}
\hline $\mathbf{R}_{\mathbf{i}}$ & $\mathbf{F D M}$ & LNS \\
\hline 0.1 & 0.22415 & 0.22304 \\
\hline 1.0 & 0.34958 & 0.34275 \\
\hline 2.0 & 0.47204 & 0.46274 \\
\hline 3.0 & 0.58357 & 0.57495 \\
\hline 4.0 & 0.68803 & 0.68240 \\
\hline 5.0 & 0.78664 & 0.78197 \\
\hline 6.0 & 0.88073 & 0.86677 \\
\hline 7.0 & 0.97108 & 0.91359 \\
\hline 8.0 & 1.14268 & 1.0936 \\
\hline
\end{tabular}
Table 3: Numerical values of coefficient of Skin friction $\operatorname{Re}_{x}^{\frac{1}{2}} C f_{x}$
values of $R_{i}$ when $P_{r}=10.0, \alpha=0.4, M=1.0$.

The effects of different values of Richardson parameter on coefficients of skin friction and rate of heat transfer are shown in Tables 3 and 4. From these tables it can be seen that the skin friction and heat transfer are both increased because of the influence of the buoyancy force. The effects of various values of Prandtl number Pr on coefficients of skin friction and rate of heat transfer are shown in Tables 5 and 6.

\begin{tabular}{|c|c|c|}
\hline $\mathbf{R}_{\mathbf{i}}$ & FDM & LNS \\
\hline 0.1 & 0.28611 & 0.28014 \\
\hline 1.0 & 0.33156 & 0.33975 \\
\hline 2.0 & 0.36669 & 0.36743 \\
\hline 3.0 & 0.39392 & 0.39214 \\
\hline 4.0 & 0.41644 & 0.41238 \\
\hline 5.0 & 0.43579 & 0.43184 \\
\hline 6.0 & 0.45286 & 0.46671 \\
\hline 7.0 & 0.46819 & 0.47347 \\
\hline 8.0 & 0.49496 & 0.50304 \\
\hline
\end{tabular}

Table 4: Numerical values of coefficient of rate of heat transfer $N u_{x} R e_{x}^{-\frac{1}{2}}$ obtained for different values of $R_{i}$ when $P_{r}=10.0, \alpha=0.4, M=1.0$.

\begin{tabular}{|c|c|c|}
\hline $\mathbf{P}_{\mathbf{r}}$ & FDM & LNS \\
\hline 0.1 & 0.48354 & 0.48201 \\
\hline 1.0 & 0.48197 & 0.48175 \\
\hline 2.0 & 0.48039 & 0.48143 \\
\hline 3.0 & 0.47898 & 0.47102 \\
\hline 4.0 & 0.47772 & 0.462240 \\
\hline 5.0 & 0.47658 & 0.46001 \\
\hline 6.0 & 0.47554 & 0.45177 \\
\hline 7.0 & 0.47458 & 0.45093 \\
\hline 8.0 & 0.47368 & 0.45016 \\
\hline
\end{tabular}
Table 5: Numerical values of coefficient of Skin friction $\operatorname{Re}_{x}^{\frac{1}{2}} C f_{x}$ obtained for
different values of $\mathrm{P}$, when $\mathrm{R}=2.0, \alpha=0.4, \mathrm{M}=1.0$.

\begin{tabular}{|c|c|c|}
\hline $\mathbf{P}_{\mathbf{r}}$ & FDM & LNS \\
\hline 0.1 & 0.10516 & 0.10404 \\
\hline 1.0 & 0.14134 & 0.14963 \\
\hline 2.0 & 0.17772 & 0.17702 \\
\hline 3.0 & 0.20992 & 0.20495 \\
\hline 4.0 & 0.23859 & 0.23209 \\
\hline 5.0 & 0.26444 & 0.25970 \\
\hline 6.0 & 0.28801 & 0.27653 \\
\hline 7.0 & 0.30973 & 0.29359 \\
\hline 8.0 & 0.32993 & 0.31936 \\
\hline
\end{tabular}

Table 6: Numerical values of coefficient of rate of heat transfer $N u_{x} R e_{x}^{-\frac{1}{2}}$ obtained for different values of $P$, when $R=2.0, \alpha=0.4, M=1.0$.

From these tables it is concluded that the component of skin friction is decreased while the component of rate of heat transfer is increased due to the reason that the product of density and thermal diffusion is decreased which enhance the rate of heat transfer in the fluid flow domain. At the end the numerical results obtained by both methods are found to be in good agreement.

\section{Conclusion}

In this article we have illustrated the effects of different parameters on some physical quantities such as skin friction, rate of heat transfer, momentum and thermal boundary layer thicknesses. The dimensionless local balance equations were solved numerically using a Finite Difference and Local Non-Similarity method by FORTRAN code. At the end, we summarize the following findings.

The algebraic decay parameter $a$ influences the free stream velocity distribution at $\mathrm{Y}=16-18$, and there is no effects of algebraic decay parameter on temperature distribution. For increasing value of Prandtl number Pr both the momentum and thermal boundary layer thicknesses are decreased with the reason that with the increase of Prandtl number Pr the fluid become more viscous which slow down the 
Citation: Ashraf M, Ahmad U, Ahmad M, Sultana N (2015) Computational Study of Mixed Convection Flow with Algebraic Decay of Mainstream Velocity in the Presence of Applied Magnetic Field. J Appl Mech Eng 4: 175. doi:10.4172/2168-9873.1000175

fluid motion, so distributions on both cases are decaying and this decay is asymptotic. With the increase of Richard parameter the momentum boundary layer thickness is decreased because the Richardson number acts like pressure gradient which take the responsibility of this physical phenomena but its effects in the case of thermal boundary layer is vice versa. With the increase of applied magnetic field momentum boundary layer is decreased and thermal boundary layer is increased for maximum value of $M=20.0$. For increasing value of Hartmann number increase the magnetic energy and decrease the kinetic energy in the fluid flow domain due to this reason both quantities are decreased. It is also noted that the skin friction and heat transfer are both increased because of the influence of the buoyancy force. It is also concluded that the component of skin friction is decreased while the component of the rate of heat transfer is increased due to the reason that the product of density and thermal diffusion is decreased which enhance the rate of heat transfer in the fluid flow domain.

\section{References}

1. Ostrach S (1952) An analysis of laminar free convective flow and heat transfe about a flat plate parallel to direction of the generating body force. NACA Technical Report 1111

2. Sparrow EM, Chess RD (1961) The effect of magnetic field on free convection heat transfer, Int J Heat Mass Transfer 3: 267-274.

3. Sparrow EM, Minkowycz WJ (1962) Buoyancy effects of horizontal boundary layer flow and heat transfer. Int J Heat Transfer 5: 505-511.

4. Gebhart B (1962) Effects of viscous dissipation in natural convection. Journal of Fluid Mechanics 14: 225-232.

5. Nachtsheim PR, Swigert P (1965) Satisfaction of the Asymptotic Boundary Conditions in Numerical Solution of the System of Non-linear Equations of Boundary Layer Type NASA TND-3004.

6. Sparrow EM, Yu HS (1971) Local non-similarity thermal boundary layer solutions, Journal of Heat Transfer 93: 328-334.

7. Llyod JR, Sparrow EM (1970) Combined forced and free convection flow on vertical surface. International Journal of Heat Mass Transfer 13: 434-438.

8. Dwivedi RN, Dube SK (1974) Oscillatory Hydromagnetic free Convection Flow Past an Infinite Vertical Flat Plate with Suction.

9. Soundalgekar VM (1976) Effects of mass transfer on free convective flow of a dissipative, incompressible fluid past an infinite vertical porous plate with suction, Proc Indian Academy of Sciences 84A: 194-203.
10. Merkin JH (1978) On solution of the boundary layer equations with algebraic decay. Journal of Fluid Mechanics 88: 309-321.

11. Schneider W (1979) A similarity solution for combined forced and free convection flow over a horizontal plate. Int J Heat Mass Transfer 22: 14011406 .

12. Chakrobarti A, Gupta AS (1979) Hydromagnetic flow heat and mass transfer over a stretching sheet. Quarterly of Applied Mathematics 33: 73-78.

13. Perdikis C (1986) Free convection and mass transfer effects on the flow past a vertical plate. Astro-Physics and Space Science 119: 295-303.

14. Wantanabe T, Pop I (1994) Thermal boundary layers in magnetohydrodynamic flow over a flat plate in the presence of a transverse magnetic field. Acta Mechanica 105: 233-238.

15. Hosain MA, Alam KCA, Rees DAS (1997) MHD Forced and free convection boundary layer flow along a vertical porous plate. Appl Mech and Eng 2: 33-51.

16. Chamkha AJ (1997) MHD Free Convection From a Vertical Plate Embedded in a Thermally Star- tified Porous Medium with Hall Effects, Appl. Math Modelling 21: 603-609.

17. Chen TS (1998) Parabolic systems: Local Non-similarity Method, Handbook of Numerical Heat Transfer, Chap. 5 Wiley, New York.

18. Mostafa A, Mahmoud A (2007) Variable viscosity effects on hydromagnetic boundary layer along a continuously moving vertical plate in the presence of radiation. Appl Math and Sciences 1: 799-814.

19. Al- Odat, MQ, Al-Azab TA (2007) Influence of chemical reaction on transient MHD free convection over a moving vertical plate, Emirates $\mathrm{J}$ for Eng Research 12: $15-21$

20. Makinde OD (2008) On MHD Boundary Layer Flow and Mass transfer Past vertical plate in a porous medium with constant heat flux. International Journal of Numerical Methods for Heat and Fluid Flow 19: 546-554.

21. Alam MS, Rehman MM, Sattar MA (2008) Effects of variable suction and thermophoresis on steady MHD combined free-forced convective heat and mass transfer flow over a semi-infinite permeable inclined plate in the presence of Thermal Radiation, International Journal of Thermal Sciences 47: 758-765.

22. Palani G, Srikanth U (2009) MHD Flow past a semi-infinite vertical plate with mass transfer, nonlinear analysis. Modeling and control 14: 345-356.

23. Ferdows M, Md Khan S, Alam Md M, Sun S (2012) MHD Mixed convective boundary layer flow of a nanofluid through a porous medium due to an exponentially stretching sheet. Mathematical Problems in Engineering 21.

24. Ashraf M, Asghar S, Hossain MA (2010) Thermal radiation effects on hydromagnetic mixed convection flow along a magnetized vertical porous plate. Mathematical Problems in Engineering 30 